\title{
Everything should be as simple as possible, but this will still be complex: A reply to various commentaries on IPEBA
}

We thank the Editor for inviting us to reply to our esteemed colleagues who shared their views on our paper, which reflects on some of the uses (not the usefulness) of the taxonomies of behaviour change techniques (Armitage, 2014; Field, 2014; Johnson \& Michie, 2006; Knittle, 2014; Michie, Johnson, \& Johnston, 2014; Peters, de Bruin, \& Crutzen, 2014). We think this has now become an exciting debate on this topic, and we hope that this reply is a useful addition to that.

Before we reply to specific comments, we would like to first reiterate the main points of our paper: The recently developed, consensus-based taxonomies of BCTs are a great resource (Abraham \& Michie, 2008; Michie et al., 2013). They have been developed to improve reporting of behaviour change interventions and to allow for the coding of the potentially active ingredients of behaviour change interventions. The current taxonomies, however, capture only a segment of what theory and evidence has to say about the 'active content' of behaviour change interventions, since they do not contain any information on mediators (i.e., the processes or determinants through with BCTs influence behaviour) and moderators (i.e., the conditions under which the BCTs have been shown, or are theorized, to be effective). This is not a critique of the usefulness of the BCT taxonomies, since this is not what they were designed for in the first place. It has, however, several important implications for how these taxonomies can be used.

First, without a theorized or evidence-based link between BCTs and behavioural determinants (or behaviour directly through a yet unknown process), it is unclear how BCT taxonomies can be used for intervention development. Second, when just relying on current BCT taxonomies without considering what theories and previous research reveal about the relevant parameters for effectiveness of BCTs (e.g., self-efficacy needs to be enhanced when using the BCT 'fear appeals' (Peters, Ruiter, \& Kok, 2013; Witte, 1992); arguments need to be new to the target group to be 'persuasive arguments' (Petty, Barden, \& Wheeler, 2009)), people developing interventions may be creating weak or even counterproductive 
interventions; reports of the active content of interventions in articles are incomplete (e.g., unclear whether parameters for effectiveness were taken into account in the intervention); and those synthesizing the evidence of behaviour change interventions will not distinguish between interventions in which the parameters for effective application of BCTs were met or not, which might lead to flawed conclusions and theorizing.

In the IPEBA paper we particularly pay attention to the potential limitations of evidence syntheses that use meta-analysis to relate BCTs to intervention effect sizes, often without considering the parameters for BCT effectiveness, BCT co-occurrence, the number of BCTs in interventions, variability in the active content of support provided to control groups, and differences in context and populations. There are fields (including one inhabited by Dr. Field, a.k.a. The Judge) where people would not consider conducting meta-analysis of studies/interventions that are as different as they tend to be in our meta-analyses (they would instead do a systematic review). Yet, in the field of health behaviour change interventions many choose to embrace this heterogeneity and run (series of) meta-analyses to explain this heterogeneity based on study characteristics such as BCTs. Whereas under certain conditions this can yield valuable results, such analyses yield observational and not experimental evidence, and given the sheer number of potential effect modifiers (or: confounders) the causality of associations is highly uncertain. This, however, does not tend to be reflected in authors' conclusions and might be misleading for people interpreting the evidence from such meta-analyses. This is well-illustrated in one of the commentaries, which makes various excellent points (to which we will return later) but phrases the results of a cited meta-analysis causally: "As combining self-monitoring with other BCTs derived from control theory (e.g., goal setting, provision of feedback, action planning) leads to greater changes in healthy eating and physical activity than other interventions (Michie, Abraham, Whittington, McAteer, \& Gupta, 2009) [...]" (Knittle, 2014, p. 2). To arrive at this conclusion, we think the meta-analysis needs to be either based on a set of studies directly testing this (i.e., control theory interventions versus 'other interventions'), or these results need to be 
corroborated by studies experimentally testing the associations observed in the metaanalysis. And this is the key point of IPEBA.

So what we propose here is to pause for a minute and reflect on how we - as a field might best tackle the relevant questions. We think it is about recognizing the strengths and limitations of evidence from meta-analyses of behaviour change interventions and from experimental studies, and strategically combining both in order to identify what interventions work, for whom, and under what conditions. To combine the excitement about the usefulness of BCT taxonomies and associations we find in meta-analyses, with a healthy dose of critical thinking about what these findings really mean. We come up with various concrete suggestions on how some limitations might be addressed, to which those commenting on our paper add a series of additional strategies and considerations, such as the use of fractional factorial designs (Armitage, 2014; Field, 2014; Johnson \& Michie, 2006; Knittle, 2014; Michie et al., 2014). We are not saying that meanwhile it isn't possible to develop interventions and advice policy makers (cf. Armitage, 2014; Michie et al., 2014); there are plenty of theories and existing evidence on BCTs, their mediators and moderators (summarized in for example the BCT taxonomy used in Intervention Mapping; Bartholomew, Parcel, Kok, \& Gottlieb, 2001; Bartholomew et al., 2006; Bartholomew, Parcel, Kok, Gottlieb, \& Fernández, 2011) to guide us (this research did not start with the 2008 Abraham and Michie taxonomy, nor with Intervention Mapping). What we do say is that in our scientific work we could be more careful in our statements, and more strategic as a community in our efforts towards advancing our evidence base on what works for whom and under what conditions. For example, if a meta-analysis of complex/multi-technique interventions finds that interventions containing self-monitoring seem to be more effective (Michie, Abraham, Whittington, McAteer, \& Gupta, 2009), as Armitage (2014) also suggests, our field could zoom in using experimental methods to identify exactly when, for whom and how this BCT can best be delivered (and, in fact, whether it can be experimentally confirmed that selfmonitoring works). 
After reading the commentaries, we felt that our position was pretty well in the middle of the opinions expressed. Somewhat ahead of all of us, The Judge (Field, 2014) raises a number of relevant statistical considerations that lead to the conclusion that systematic reviews might be more appropriate for synthesizing the evidence of behavioural interventions than meta-regression analyses; and that in order to identify which BCTs work and under what conditions, experiments directly manipulating these elements seem to be most suitable. Knittle (2014) is absolutely right about complicating the situation further, by raising the issue of intervention fidelity. This is rarely reported (especially at the BCT level) or coded for in reviews of complex behaviour change interventions, yet we all know intervention fidelity can be a major issue. This further limits what we can infer from meta-analyses linking adequately reported and reliable coded (as opposed to actually delivered) BCTs to effect sizes. The compelling points raised by Johnson and Michie (2014) seem to be in agreement with the points we make, at least for those we thought were within the realm of IPEBA. That is, point 3 describes what we would call parameters for effectiveness (Peters et al., 2014, p. 3) and confounders (Peters et al., 2014, p. 4). As Johnson and Michie argue, these can also be based on insights from allied fields (e.g., sociology, anthropology, behavioural economics). Point 4 is in line with our suggestions for improving the quality of meta-analysis. Finally, Michie, Johnson, and Johnston (2014) partly agree with some of the points we made, refer to several additional designs and statistical procedures that can be used to more successfully identify effective BCTs (although each has its own limitations again), and refer to several studies that are not suffering from some of the limitations we discussed. Naturally, our comments on current practices do not mean that we think nobody is doing 'the right thing' (just as the commentary authors, when stating that the BCT taxonomy should be used for reporting of interventions, are probably not meaning to say that nobody is reporting their intervention properly).

In sum, the reason we wrote this IPEBA paper is because we think that when designing behaviour change interventions, and when synthesizing their evidence with the purpose of identifying active ingredients, it is important to consider what theory and prior studies says 
about mediators (e.g., behavioural determinants) and moderators (e.g., parameters of BCT effectiveness). Moreover, regularly too strong conclusions seem to be drawn from metaanalyses linking BCTs to intervention effects. Finally, we think there is an opportunity for shaping our research practice as a community in a way that may lead to a more rapid progression of scientific evidence and behaviour change theory (cf. de Bruin \& Johnston, 2012; Platt, 1964). The commentaries made valuable suggestions for additional elements to be considered, including references to exemplary studies, but also seemed to disagree on whether meta-analyses are appropriate methods for this purpose. We think they are, given certain quality criteria described in this paper; but we would like to strongly argue for a more inquisitive attitude towards the validity of the results generated by these meta-analyses, and a more systematic approach in the field of combining methodologies for identifying what BCTs work, how, for whom, and under what conditions.

\section{References}

Abraham, C., \& Michie, S. (2008). A taxonomy of behavior change techniques used in interventions. Health Psychology, 27, 379-387.

Armitage, C. J. (2014). Changing behaviour, slow and fast: commentary on Peters, de Bruin and Crutzen. Health Psychology Review, (May), 1-4. doi:10.1080/17437199.2014.912537

Bartholomew, L. K., Parcel, G. S., Kok, G., \& Gottlieb, N. H. (2001). Intervention mapping: designing theory and evidence-based health promotion programs. Mountain View, CA: Mayfield publishing company.

Bartholomew, L. K., Parcel, G. S., Kok, G., Gottlieb, N. H., \& Fernández, M. E. (2011). Planning health promotion programs: an Intervention Mapping approach (3rd ed.). San Francisco, CA: Jossey-Bass. 
Bartholomew, L. K., Parcel, G. S., Kok, G., Gottlieb, N. H., Intervention, A., \& Approach, M. (2006). Intervention mapping: designing theory and evidence-based health promotion programs. San Francisco, CA: Jossey-Bass.

De Bruin, M., \& Johnston, M. (2012). Methods in health psychology: how do we know what we really know? The European Health Psychologist, 14(4), 107-112.

Field, A. P. (2014). Dread returns to Mega-Silly One. Health Psychology Review. doi:10.1080/17437199.2013.879198

Johnson, B. T., \& Michie, S. (2006). Towards healthy theorising about health behaviours in the maze of messy reality : a reaction to Peters, de Bruin , and Crutzen, (May 2014), 37-41. doi:10.1080/17437199.2014.900722

Johnson, B. T., \& Michie, S. (2014). Towards healthy theorising about health behaviours in the maze of messy reality: a reaction to Peters, de Bruin, and Crutzen. Health Psychology Review, 1-4. doi:10.1080/17437199.2014.900722

Knittle, K. (2014). We cannot keep firing blanks - yet another appeal for improved rct reporting: commentary on Peters, de Bruin \& Crutzen. Health Psychology Review, (March), 1-5. doi:10.1080/17437199.2014.900721

Michie, S., Abraham, C., Whittington, C., McAteer, J., \& Gupta, S. (2009). Effective techniques in healthy eating and physical activity interventions: a meta-regression. Health Psychology, 28(6), 690-701. doi:10.1037/a0016136

Michie, S., Johnson, B. T., \& Johnston, M. (2014). Advancing cumulative evidence on behaviour change techniques and interventions: a comment on Peters, de Bruin, and Crutzen. Health Psychology Review. doi:10.1080/17437199.2014.912538

Michie, S., Richardson, M., Johnston, M., Abraham, C., Francis, J., Hardeman, W., ... Wood, C. E. (2013). The Behavior Change Technique Taxonomy (v1) of 93 Hierarchically Clustered Techniques: Building an International Consensus for the Reporting of Behavior Change Interventions. Annals of Behavioral Medicine : A Publication of the Society of Behavioral Medicine. doi:10.1007/s12160-013-9486-6 
Peters, G.-J. Y., de Bruin, M., \& Crutzen, R. (2014). Everything should be as simple as possible, but no simpler: towards a protocol for accumulating evidence regarding the active content of health behaviour change interventions. Health Psychology Review, 114. doi:10.1080/17437199.2013.848409

Peters, G.-J. Y., Ruiter, R. A. C., \& Kok, G. (2013). Threatening communication: a critical reanalysis and a revised meta-analytic test of fear appeal theory. Health Psychology Review, 7(Suppl 1), S8-S31. doi:10.1080/17437199.2012.703527

Petty, R. E., Barden, J., \& Wheeler, S. C. (2009). The Elaboration Likelihood Model of Persuasion: Developing Health promotions for Sustained Behavioral Change. In R. J. DiClemente, R. A. Crosby, \& M. Kegler (Eds.), Emerging theories in health promotion practice and research (2nd ed., pp. 185-214). San Francisco: Jossey-Bass.

Platt, J. R. (1964). Strong Inference. Science, 146(3642), 347-353.

Witte, K. (1992). Putting the fear back into fear appeals: The extended parallel process model. Communication Monographs, 59(4), 329-349.

doi:10.1080/03637759209376276 